\title{
Correction to: Semi-analytical prices for lookback and barrier options under the Heston model
}

\author{
Luca De Gennaro Aquino ${ }^{1} \cdot$ Carole Bernard ${ }^{1,2}$ (i)
}

Accepted: 1 October 2021 / Published online: 27 October 2021

(C) The Author(s), under exclusive licence to Associazione per la Matematica Applicata alle Scienze Economiche e Sociali (AMASES) 2021

\begin{abstract}
In this note, we point out a mistake in Theorem 1 of De De Gennaro Aquino and Bernard (Decis Econ Finance 42(2):715-741, 2019) and provide some missing references where the problem of pricing barrier options under the Heston model had previously been discussed.
\end{abstract}

Keywords Derivatives pricing · Lookback options · Barrier options · Path-dependent options $\cdot$ Heston model $\cdot$ Stochastic volatility

JEL Classification C65 - G13

\section{Correction to: Decisions in Economics and Finance (2019) 42:715-741 https://doi.org/10.1007/s10203-019-00254-X}

\section{Discussion}

There is a mistake in the proof of Theorem 1 in De Gennaro Aquino and Bernard (2019). Specifically, this mistake has roots in Subsection 2.2. Therein, we write that under the Heston stochastic volatility model, the spot price at maturity $T$ can be written

The original article can be found online at https://doi.org/10.1007/s10203-019-00254-x.

Luca De Gennaro Aquino

luca.degennaroaquino@grenoble-em.com

Carole Bernard

carole.bernard@grenoble-em.com

1 Department of Accounting, Law and Finance, Grenoble Ecole de Management, 12 Rue Pierre Sémard, 38000 Grenoble, France

2 Department of Economics and Political Sciences, Vrije Universiteit Brussel (VUB), Brussel, Belgium 
as

$$
S_{T}=S_{0} e^{r T} \exp \left(\sqrt{1-\rho^{2}} \int_{0}^{T} \sqrt{v_{s}} d W_{s}+\mu_{v}\right),
$$

where $\mu_{v}$ is given by

$$
\mu_{v}:=r T-\frac{1}{2} \int_{0}^{T} v_{s} d s+\frac{\rho}{\sigma}\left(v_{T}-v_{0}-\kappa \theta T+\kappa \int_{0}^{T} v_{s} d s\right) .
$$

Then, we observe that after conditioning with respect to the filtration generated by the variance process $\left\{v_{t}\right\}_{0 \leq t \leq T}$, i.e., $\mathcal{F}_{t}^{v}=\sigma\left(\left\{v_{s}: 0 \leq s \leq t\right\}\right), \mu_{v}$ may be interpreted as a deterministic quantity and the stock price $S_{T}$ defined by (1) has a (conditional) lognormal distribution.

While the latter observation is not inherently incorrect if one needs to examine pathindependent payoffs (that is, payoffs depending only on the stock price at maturity), the same argument cannot be relied upon for path-dependent payoffs, which is the case of our paper. In essence, one can show that it leads to a description of the underlying stock price where the variance process is erroneously averaged.

This error thus invalidates the statement of Theorem 1, where we derive the joint density of the logarithmic stock price at maturity and its maximum, conditional on $\mathcal{F}^{v}$, and the joint density of the logarithmic stock price at maturity and its minimum, yet conditional on $\mathcal{F}^{v}{ }^{1}$ In light of this issue, and for Theorem 1 being a key ingredient to derive the pricing formulas for lookback, partial lookback and barrier options under the Heston model in Sections 3, 4 and 5, respectively, all semi-analytical formulas provided in our paper do not give exact prices, but only (non-convergent) approximations of them.

A final comment. Concurrently to the letter pointing out the above-mentioned mistake, we were further communicated that the problem of pricing barrier options under the Heston model had previously been discussed in Lipton (2001) and Lipton and McGhee (2002). Regrettably, we were not aware of these studies at the time and thus apologize to the authors for the omission.

Acknowledgements The authors wish to thank Alexander Lipton for pointing out the mistake in the paper and providing helpful discussions.

\section{References}

De Gennaro Aquino, L., Bernard, C.: Semi-analytical prices for lookback and barrier options under the Heston model. Decis Econ Finance 42(2), 715-741 (2019)

Lipton, A.: Mathematical Methods for Foreign Exchange: A Financial Engineer's Approach. World Scientific (2001)

\footnotetext{
1 The theorem is flawed in the sense that it does not provide the true conditional joint density of the logarithmic spot price and its maximum, or minimum, under the Heston model. For reference only, the expressions in Eqs. (9), (11) and (12) remain valid if all involved coefficients are indeed (unconditionally) constant.
} 
Lipton, A., McGhee, W.: Universal barriers. Risk 15(5), 81-85 (2002)

Publisher's Note Springer Nature remains neutral with regard to jurisdictional claims in published maps and institutional affiliations. 